\title{
DISKUSSION
}

\section{Direktwahl der Ministerpräsidenten? Eine Entgegnung auf Everhard Holtmanns Replik in Heft 1/2011 der ZParl}

\author{
Frank Decker
}

\begin{abstract}
„Die sechzehn Bundesländer brauchen nicht notwendig Regierung und Opposition; vielmehr ist ihnen eine anständige Verwaltung notwendig und ebenso ein Landtag, der die Verwaltung sorgfältig überwacht. Das Problem der Koalitionsbildung stellt sich dagegen im Bund, denn

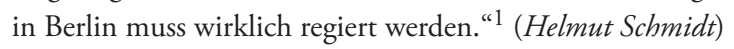

Dass mein in Heft 3/2010 der ZParl gemachter Vorschlag, die parlamentarische Regierungsform in den Ländern durch Einführung der Direktwahl der Ministerpräsidenten ${ }^{2}$ zu überwinden ${ }^{3}$, Widerspruch auslösen würde, stellt keine Überraschung dar. Everhard Holtmanns Replik ${ }^{4}$ enthält sich erfreulicherweise jeder Schärfe oder Polemik. Die von ihm vorgetragenen Bedenken münden nicht in eine rigorose Zurückweisung der Reformüberlegungen. Indem sie - nüchtern abwägend - auf institutionelle Folgewirkungen und mögliche Dysfunktionalitäten hinweisen, wollen sie vielmehr vor übertriebenen oder falschen Erwartungen warnen, die in den Vorschlag gesetzt werden. Holtmanns Plädoyer, dass eine Reorganisation der Regierungssysteme in den Ländern einer sorgfältigen „präventiven Folgenabschätzung“ bedarf, ist uneingeschränkt zuzustimmen. Auch seine Gegenargumente, die sich unter anderem auf die Erfahrungen mit den präsidentialisierten Regierungssystemen auf kommunaler Ebene stützen, sind durchweg fundiert. Der Unterschied reduziert sich insofern darauf, dass ich in der Abwägung der Pro- und Kontra-Gesichtspunkte zu anderen Schlussfolgerungen komme.

Zunächst ist auf ein Kuriosum hinzuweisen. Holtmanns Replik bezieht sich auf einen Vorschlag, der gar nicht das Kernthema meines ursprünglichen Aufsatzes war, sondern im Rahmen meiner Überlegungen zur „Systemverträglichkeit“ der in den Ländern bestehenden Verfahren der direkten Demokratie nur beiläufig erwähnt wurde. Die darauf bezogenen Ausführungen umfassen gerade einmal eine halbe Seite. Sie erörtern die Direktwahl nicht in Gänze, sondern einzig im Blick auf ihre - von mir behauptete - bessere Kompatibilität mit der Volksgesetzgebung. Über diesen, in der Literatur bisher kaum beachteten Punkt geht Holtmann leider rasch hinweg, während er gleichzeitig die vom Autor an anderer Stelle veröffentlichten Beiträge zur Direktwahl unberücksichtigt lässt, die sich mit dem Thema inten-

1 Helmut Schmidt im Interview mit Giovanni di Lorenzo, Auf eine Zigarette mit Helmut Schmidt - Über Koalitionen und italienische Zustände, in: Zeit Magazin vom 21. Februar 2008, S. 54.

2 Der Begriff Ministerpräsident steht hier synonym für die Regierungschefs in den Ländern, umfasst also auch die Bürgermeister der Stadtstaaten. Dasselbe gilt für die als Landtag bezeichneten Parlamente, die in Bremen und Hamburg Bürgerschaft, in Berlin Abgeordnetenhaus heißen.

3 Vgl. Frank Decker, Zwischen Placebo und Erfolgsmodell. Direkte Demokratie auf der Landesebene, in: ZParl, 41. Jg. (2010), H. 3, S. $564-579$.

4 Vgl. Everhard Holtmann, Direkt gewählte Ministerpräsidenten der Länder - eine kritische Folgenabschätzung der von Frank Decker in Heft 3/2010 der ZParl veröffentlichten Überlegungen, in: ZParl, 42. Jg. (2011), H. 1, S. $194-205$. 
siver befassen. Sie werden jedenfalls - genauso wie andere einschlägige Pro-Stimmen in den Anmerkungen nicht erwähnt. ${ }^{5}$ Dies ist insofern misslich, als dort manche der von Holtmann formulierten Einwände bereits aufgenommen und auf ihre Triftigkeit überprüft wurden; der eine oder andere Kritikpunkt hätte sich damit vielleicht erübrigt.

Holtmann unterstellt in einem „kategorialen Vorbehalt“, dass ich Volksgesetzgebung und Direktwahl in dieselbe „plebiszitäre“ Schublade packen würde. ${ }^{6}$ Dies ist in doppelter Hinsicht unzutreffend. Zum einen habe ich in früheren Publikationen ausdrücklich dafür geworben, die Direktwahl gerade nicht unter die direktdemokratischen Verfahren zu subsumieren. Letztere umfassen aus meiner Sicht nur Abstimmungen, die außerhalb der regulären Wahlen stattfinden. ${ }^{7}$ Zum anderen mache ich mir auch die Argumentation von Autoren wie Hans Herbert von Arnim nicht zueigen, die in der Volksgesetzgebung und Direktwahl gleichgerichtete Möglichkeiten sehen, die vermeintliche Allmacht der Parteien in unserem politischen System zu brechen. Insofern fällt der Vorwurf der „,begrifflich-systematischen Unschärfe" auf Holtmann selbst zurück, wenn er Volksgesetzgebung und Direktwahl mit dem Hinweis kritisiert, beide Verfahren schwächten die in der repräsentativen Demokratie notwendige Integrationsfunktion der Parteien. ${ }^{8}$ Mir kam es auf einen anderen, „ursprünglicheren“ Zusammenhang an: Die Volksgesetzgebung stellt danach wie die Direktwahl ein Element der Gewaltentrennung dar, das der gewaltenfusionierenden Funktionslogik des parlamentarischen Systems widerspricht. Weil die Möglichkeit, diesen Widerspruch in den Ländern durch eine Abschaffung oder starke Beschneidung der Volksrechte zu beseitigen, in der gegebenen Verfassungslage kaum noch besteht - allein der Versuch würde erheblichen Widerstand auslösen -, kommt die Abkehr von der parlamentarischen Regierungsform als alternativer Reformpfad ins Spiel.

Gegen die von mir konstatierte größere Affinität der Landespolitik zur Kommunal- als zur Bundesebene betont Holtmann zu Recht, dass sich die Strukturen der kommunalen Regierungssysteme nicht eins-zu-eins auf die Länder übertragen lassen. Damit würde die in der vergleichenden Forschung hinlänglich beschriebene Schwäche des Präsidentialismus dort automatisch stärker hervortreten: die Schwierigkeiten, dauerhaft tragfähige legislative Mehrheiten zu bilden, um ein konsistentes, auf innovative Problemlösungen abzielendes Regieren zu gewährleisten. Solche Schwierigkeiten sind vor allem (aber nicht nur) unter den Bedingungen eines divided government zu erwarten, wenn Ministerpräsident und Landtagsmehrheit verschiedenen parteipolitischen Lagern entstammen.

Das Blockadeargument lässt sich schon aufgrund der starken Überlagerung der Landesdurch die Bundespolitik nicht von der Hand weisen. Ein taugliches Präsidentialismusmodell für die Länder dürfte insofern eher in Richtung eines „präsidentiell-parlamentarischen“ Mischsystems tendieren. Es müsste durch geeignete institutionelle Vorkehrungen für einen

5 Vgl. insbesondere Frank Decker, Direktwahl der Ministerpräsidenten?, in: Recht und Politik, 37. Jg. (2001), H. 3, S. 152 - 161. Die beste Gesamtdarstellung des Vorschlags im Für und Wider findet sich in einer von Hans Herbert von Arnim betreuten Speyerer Dissertation. Vgl. Jan L. Bachmann, Direktwahl der Ministerpräsidenten. Als Kern einer Reform der Landesverfassungen, Berlin 2006.

6 Vgl. Everhard Holtmann, a.a.O. (Fn. 4), S. 194.

7 Neben den Abstimmungen über Sachfragen gehören danach auch personenbezogene Abstimmungen wie der „recall“ zu den plebiszitären Elementen. Zur Begriffsdiskussion vgl. Frank Decker, Präsidialsystem und direkte Demokratie in der Europäischen Union?, in: Hans Herbert von Arnim (Hrsg.), Reform der Parteiendemokratie, Berlin 2003, S. 79 - 95.

8 Vgl. Everhard Holtmann, a.a.O. (Fn. 4), S. 195, S. 204. 
annähernden politischen Gleichklang zwischen Exekutive und Legislative sorgen. Der direkt gewählte Regierungschef bliebe so in den Willen der jeweiligen Parlamentsmehrheit eingebunden. Die Anbindung könnte zum einen dadurch erfolgen, dass der Landtag das Recht behält, die vom Ministerpräsidenten ernannten Regierungsmitglieder vor ihrem Amtsantritt zu bestätigen. ${ }^{9}$ Zum anderen sollte der Regierungschef, wie beim kommunalen recall heute schon der Fall, auch aus politischen Gründen abberufen werden können - allerdings unter höheren Hürden als im normalen parlamentarischen System. Vorstellbar wären zum Beispiel ein spezielles Quorum und / oder eine automatische Verknüpfung von Abberufung und Parlamentsauflösung (mit anschließenden Neuwahlen).

Auf die institutionelle Ausgestaltung des Direktwahlvorschlags geht Holtmann nicht näher ein. Sein Hauptaugenmerk gebührt in diesem Zusammenhang den Negativwirkungen, die von einer Abschaffung der Sperrklauseln im Landtagswahlrecht ausgehen würden. In der Tat ist nicht einzusehen, warum die Landesverfassungsgerichte diese nur im Kontext einer parlamentarischen Regierungsform für legitimierbar halten, so als ob sie für die Gewährleistung von Regierungsfähigkeit im präsidentiellen System keine Rolle spielten. Wenn von Teilen der Wissenschaft heute die Wiedereinführung beziehungsweise Anhebung der Sperrklauseln auf kommunaler Ebene ins Spiel gebracht wird, um einer übermäßigen Fragmentierung der parlamentarischen Vertretungen entgegenzuwirken, muss das erst recht für die Länder gelten.

Weniger einleuchtend ist Holtmanns Argumentation mit Blick auf das Stimmrecht im Bundesrat. Sein Hinweis auf mögliche Koppelgeschäfte zwischen Regierung und Landtagsmehrheit übersieht, dass den Landtagen die Mitwirkung an der Bundesgesetzgebung im deutschen Exekutivföderalismus ja bereits heute generell versagt bleibt, und zwar nicht nur de jure, indem sie den Regierungen keine rechtsverbindlichen Weisungen erteilen dürfen, sondern auch faktisch. ${ }^{10}$ Die Einführung der Direktwahl würde daran nichts ändern. Sie wäre im Gegenteil - wie Holtmann selbst andeutet ${ }^{11}$ - gerade mit Blick auf den heutigen Enthaltungsautomatismus der „gemischten“ Koalitionen heilsam, da die Landesregierungen auf diese Weise angehalten werden, sich auf ihr Stimmverhalten politisch zu verständigen. Weniger verlässlich prognostizierbar ist, wie sich ein „monokratisches“ Ministerpräsidentenregime im Verhältnis von Regierungs- und Bundesratsmehrheit auswirken würde. Die Befürworter des Direktwahlvorschlags erwarten eine Verminderung möglicher Blockaden, weil ein direkt gewählter Regierungschef noch stärker als sein parlamentarisch bestellter Kollege dem Landesinteresse verpflichtet wäre und somit unabhängiger von der Bundespartei agieren könnte. Die Kritiker hegen dagegen die Befürchtung, dass bei einem Wegfall der parlamentarischen Koalitionsregierungen in den Ländern die Gegensätze zwischen Regierung und Opposition im Bundesrat unvermittelter aufeinanderprallen und die Kompromissbildung eher erschweren würden. Dabei gehen sie zu Recht davon aus, dass der Zwischenwahleffekt der heutigen Landtagswahlen, der die gegenläufigen Mehrheiten erzeugt, auch bei den Direktwahlen aufträte.

9 In Theodor Eschenburgs nicht ausgeführtem Verfassungsentwurf für das 1952 neu geschaffene Land Baden-Württemberg war sogar eine förmliche Wahl der Minister durch das Parlament vorgesehen; dies hätte in die Nähe der schweizerischen Kollegialregierungen geführt. Vgl. ders., Verfassung und Verwaltungsaufbau des Südweststaates, Stuttgart 1952, S. 66 f.

10 Vgl. Winfried Steffani, Bund und Länder in der Bundesrepublik, in: Falk Esche / Jürgen Hartmann (Hrsg.), Handbuch der deutschen Bundesländer, Frankfurt am Main / New York 1990, S. 37 51, S. 50.

11 Vgl. Everhard Holtmann, a.a.O. (Fn. 4), S. 202. 
Holtmann nimmt an, dass die Verschiebung von Zuständigkeiten, die ein Wechsel zur präsidentiellen Regierungsform nach sich zieht, den Regeln eines Nullsummenspiels unterliegt: „Was die Exekutive gewinnt, verliert der Landtag, und umgekehrt." ${ }^{2}$ Damit verkennt er aber die eigentliche Intention der Reform, die gerade darin besteht, die Parlamente in ihrer Gesetzgebungs- und Kontrollfunktion aufzuwerten. Dies wird im übrigen auch von Holtmann selbst eingeräumt, wenn er schreibt, dass ein präsidentialisiertes Regierungssystem den Aufbau einer „parlamentsseitigen Gegenbürokratie“ erforderlich machen würde. Dass diese nicht kostenneutral zu haben sei, ist richtig und könnte der Begeisterung der Bevölkerung für die Direktwahl, wenn es an deren Umsetzung geht, gewiss einen Dämpfer versetzen. Auf der anderen Seite kann es aber nicht angehen, dass sinnvolle Demokratiereformen an solchen Kostengesichtspunkten scheitern. Wer sich über den Bedeutungsverlust der Parlamente (nicht nur auf der Landesebene) ständig beklagt, muss auch bereit sein, in deren Stärkung zu investieren.

Holtmann stellt in seiner Analyse vor allem auf die Outputseite des Regierens ab, auf Kriterien der Funktionsfähigkeit und Effizienz. Er argumentiert also aus einer gouvernementalen Perspektive heraus, während bei mir demokratiepolitische Überlegungen im Zentrum stehen. Holtmanns Plädoyer für ein möglichst störunanfälliges Regierungssystem könnte man den zugegebenermaßen etwas flapsigen Hinweis entgegensetzen, dass der demokratische Prozess nun einmal störanfällig ist. Dies bedeutet nicht, dass das präsidentielle System unter Demokratiegesichtspunkten per se vorzugswürdig wäre. Seine Funktionsprobleme fallen aber auf der Landesebene, wo die Gesetzgebungstätigkeit gegenüber den Verwaltungsaufgaben quantitativ wie qualitativ in den Hintergrund tritt, vergleichsweise wenig ins Gewicht und werden durch die systemischen Vorteile allemal aufgewogen. Auf der nationalen Ebene steht das parlamentarische System dagegen nicht zur Disposition. Weil im Bund, wie Altkanzler Helmut Schmidt treffend sagt „wirklich regiert werden muss“, kann es seine Stärken hier voll ausspielen.

Ein Wechsel zum präsidentiellen System in den Ländern wäre nicht nur für deren politischen Systeme selbst von Nutzen. Er käme dem Föderalismus insgesamt zugute, weil er dazu beitragen würde, die notorische Überlagerung der Landes- durch die Bundespolitik wenigstens etwas zurückzudrängen. Diese Überlagerung hat nicht nur mit der engen Verflechtung der beiden Ebenen zu tun; sie stellt auch eine Konsequenz der Gleichförmigkeit der Regierungssysteme in Bund und Ländern dar. Welche absurden Konsequenzen das haben kann, zeigte sich zum Beispiel nach der Landtagswahl in Hessen 2008, als die Republik über mehrere Monate von der Debatte in Atem gehalten wurde, ob die SPD-Spitzenkandidatin Andrea Ypsilanti sich mithilfe der Linken zur Ministerpräsidentin wählen lassen dürfe. Dass eine Mehrheitsbildung unter präsidentiellen Vorzeichen ähnliche Aufmerksamkeit auf sich ziehen könnte, erscheint kaum vorstellbar. Eine Abkehr der Gliedstaaten von der parlamentarischen Regierungsform des Bundes hätte also zur Folge, dass sie die Eigenständigkeit der Landespolitik - unabhängig von der föderalen Aufgabenverteilung - automatisch stärker hervorheben würde.

Holtmanns Sorge vor einem „Kamineffekt“, der die präsidentialisierten Landesregierungssysteme zu einem Vorbild auch für den Bund machen würde, halte ich in diesem Zusammenhang für unbegründet - dazu ist das parlamentarische System in unserer Verfassungstra- 
dition zu stark verankert. Tatsächlich war die Einflussrichtung in der Vergangenheit ja umgekehrt, indem sich die Länder im Zuge von Verfassungsreformen bei der Ausgestaltung des parlamentarischen Systems und beim Wahlrecht einerseits dem Modell des Bundes zunehmend angepasst haben. ${ }^{13}$ Auf der anderen Seite weichen sie vom grundgesetzlichen Regierungssystem weiter in wesentlichen Bereichen ab; so kennen die Landesverfassungen weder ein separates Staatsoberhaupt noch Zweite Kammern, dafür aber die direktdemokratischen Verfahren (in Gestalt der Volksgesetzgebung). Die Sorge vor einem Kamineffekt scheint mir heute vor allem beim letzten Punkt angebracht, denn so wie das Vorhandensein der Volksgesetzgebung in den Ländern Zweifel an der Sinnhaftigkeit der dortigen parlamentarischen Regierungsform weckt, so weckt das vorhandene parlamentarische Regierungssystem im Bund Zweifel an der Sinnhaftigkeit einer dort einzuführenden Volksgesetzgebung.

Damit bleibt die Frage, ob die mit dieser Entgegnung fortgeführte Kontroverse nicht ohnehin müßig ist, weil es keine realistischen Chancen gibt, die Direktwahl in absehbarer Zeit einzuführen. Bis vor einigen Jahren hätte man darauf mit einem klaren Ja antworten müssen. Dass das heute nicht mehr geht, liegt wiederum an den Einrichtungen der direkten Demokratie. So wie die Bürger mit deren Hilfe Wahlrechtsreformen und erleichterte Anwendungsbedingungen für die Plebiszite zum Teil gegen den Widerstand der Regierenden durchgesetzt haben, so könnten sie als Gesetzgeber irgendwann auch die parlamentarische Regierungsform ins Visier nehmen. Diese, vom Verfasser schon früher geäußerte Vermutung ${ }^{14}$, scheint sich inzwischen zu bewahrheiten, nachdem die Ökologisch-Demokratische Partei (ÖDP) in Bayern, ein Volksbegehren für die Einführung der Direktwahl anstrengt. ${ }^{15}$ Der Freistaat wäre für einen solchen Vorstoß in der Tat prädestiniert. Zum einen steht dort mit der Nominierung des populären Münchener Oberbürgermeisters Christian Ude als SPD-Spitzenkandidat bei der kommenden Landtagswahl ein äußerst spannendes Rennen um die Regierungsmacht bevor, das durch die Direktwahlforderung zusätzliche Brisanz erlangen würde. Zum anderen haben die Bayern mit einer unangenehmen Begleiterscheinung des parlamentarischen Systems zuletzt gleich zweimal Bekanntschaft gemacht: 2007 wurde CSU-Ministerpräsident Edmund Stoiber während der laufenden Wahlperiode von seiner Partei gestürzt, für die er bei der Landtagswahl 2003 noch ein Rekordwahlergebnis erzielt hatte. Und 2008, als Horst Seehofer den wegen der hohen CSU-Verluste zurückgetretenen Stoiber-Nachfolger Günther Beckstein kurzerhand ersetzte, erhielt das Land einen Regierungschef, der bei der Wahl als Spitzenkandidat gar nicht angetreten war.

Sollte der Wechsel zum präsidentiellen System in den Ländern tatsächlich in den Bereich des Möglichen rücken, würde es sich rächen, dass die Politikwissenschaft (wie auch die Staatsrechtslehre) dieses Thema in den letzten Jahren sträflich vernachlässigt hat. Weil es stimmt, dass die Pro- und Kontra-Argumente einer solchen Reform gründlich gegeneinander abgewogen werden müssen, könnten Everhard Holtmanns Diskussionsbeitrag und meine Entgegnung vielleicht den Auftakt zu einer breiter angelegten Debatte bilden.

13 Vgl. Frank Decker, Die Regierungssysteme in den Ländern, in: ders. (Hrsg.), Föderalismus an der Wegscheide?, Wiesbaden 2004, S. 169 - 201, S. $171 \mathrm{ff}$.

14 Vgl. ebenda, S. 197 f.

15 Landesvorstand und Landeshauptausschuss der ÖDP haben festgelegt, die Unterschriftensammlung Anfang 2012 zu beginnen. Mit der Initiative soll Artikel 44 der bayerischen Verfassung geändert werden, der bislang vorgibt, dass der Landtag den Ministerpräsidenten wählt. 